\title{
Einsatzstelle Bibliothek
}

\section{Vielfalt und Engagementorientierung in den Freiwilligendiensten Kultur und Bildung}

https://doi.org/10.1515/bfp-2020-0104

Zusammenfassung: Die Bundesvereinigung Kulturelle Kinder- und Jugendbildung (BKJ) bietet jährlich 2500 Freiwilligen einen Einsatzplatz in den Freiwilligendiensten Kultur und Bildung. Die BKJ arbeitet dafür mit Organisationen der Kultur und Bildungsarbeit, darunter 180 Bibliotheken, in ganz Deutschland zusammen. Zentrale Prinzipien der Freiwilligendienste, wie die Schaffung inklusiver Zugänge sowie die Orientierung an den Interessen der Freiwilligen, werden vorgestellt und die Bedeutung der Einsatzstellen- und Trägerarbeit auf der Basis empirischer Daten verdeutlicht.

Schlüsselwörter: Engagement; Freiwilligendienst; FSJ Kultur; Kulturelle Bildung

\section{Library as a Place of Assignment in Voluntary Services Diversity and Orientation on Civic Involvement in Voluntary Services in the Field of Culture and Education}

Abstract: The Federal Association for Cultural Child and Youth Education (Bundesvereinigung Kulturelle Kinderund Jugendbildung, BKJ) offers 2,500 volunteers a place in the voluntary services for culture and education every year. To this end, the BKJ cooperates with organisations involved in cultural and educational work, including 180 libraries, throughout Germany. Central principles of the voluntary services, such as the creation of inclusive access and the orientation towards the interests of the volunteers, are presented and the importance of the work of the placement centres and organisations is illustrated on the basis of empirical data.

Keywords: Voluntary services in the field of culture and education; commitment; civic involvement; Voluntary Social Year; cultural education

\section{Inhalt}

1 Einleitung.................... 26

1.1 Tätigkeitsbereiche der Freiwilligen in Bibliotheken ................... 27

2 Inklusion und Diversität in den Freiwilligendiensten Kultur und Bildung . . . . . . . . . . . . 28

2.1 Vielseitige Aufgabenpakete .......... 30

2.2 Transparenz von Zugänglichkeiten und Barrieren ................. 30

2.3 Faires Vermittlungsverfahren für alle Interessierten ................... 31

2.4 Zielgerichtete Öffentlichkeitsarbeit für Interessierte vor Ort . . . . . . . . . . . . . 32

3 Mitsprache und Mitbestimmung in den Freiwilligendiensten Kultur und Bildung. . . . . . . . . . 32

3.1 Eigene Projekte der Freiwilligen als Ausdruck für Selbstbestimmung ............... 34

4 Engagementorientierung in den Freiwilligendiensten Kultur und Bildung . . . . . . . . . . . . 34

5 Trägerarbeit und Ausblick. . . . . . . . . . . . 35

\section{Einleitung}

Freiwilligendienste sind eine besondere Form des freiwilligen Engagements. Besonders sind sie deshalb, weil die Freiwilligen sich für eine bestimmte Dienstzeit von meist einem Jahr verpflichten, eine vereinbarte Anzahl von Bildungstagen besuchen sowie eine pauschale Aufwandsentschädigung pro Monat für ihre Tätigkeit erhalten (Taschengeld). Freiwilligendienste sind ein Angebot für Menschen, die die Zeit für sich nutzen und gleichzeitig etwas für Andere bzw. für die Gemeinschaft tun wollen. Ein Freiwilligendienst verknüpft freiwilliges Engagement mit der Möglichkeit, Erfahrungen zu sammeln, Orientierung erhalten $\mathrm{zu}$ können und sich persönlich weiterzuentwickeln. ${ }^{1}$

Die Bundesvereinigung Kulturelle Kinder- und Jugendbildung $(\mathrm{BKJ})^{2}$ startete 2001 das FSJ Kultur - einen

1 Vgl. BMFSFJ (2015).

2 Die Bundesvereinigung Kulturelle Kinder- und Jugendbildung (BKJ) ist der Dachverband für Kulturelle Bildung in Deutschland. Über 50 bundesweit agierende Fachorganisationen und Landesdachver- 
Jugendfreiwilligendienst speziell im Bereich Kultur. Im Laufe der letzten 20 Jahre erweiterte sich das Spektrum der Freiwilligendienste der BKJ um weitere Bereiche wie z.B. Politik und Schule sowie um Formate für Personen ab 27 Jahre. Heute absolvieren im Trägerverbund Freiwilligendienste Kultur und Bildung unter dem Dach der BKJ etwa 2500 Freiwillige einen Freiwilligendienst. Die Zufriedenheit der Freiwilligen mit ihrer Tätigkeit und ihrem Freiwilligendienst ist sehr hoch: Etwa 90 Prozent der ehemaligen Freiwilligen würden anderen Menschen einen Freiwilligendienst im Trägerverbund Freiwilligendienste Kultur und Bildung empfehlen. ${ }^{3}$

Die Trägerorganisationen unter dem Dach der BKJ arbeiten mit Organisationen der Kultur und Bildungsarbeit in ganz Deutschland zusammen. Diese Einsatzstellen umfassen das Spektrum aller künstlerischen Sparten sowie verschiedener (kultur-)pädagogischer Angebote in den Bereichen der Kultur- und Bildungsarbeit. Bibliotheken gehören ganz selbstverständlich dazu. Im Trägerverbund Freiwilligendienste Kultur und Bildung bieten derzeit 180 Bibliotheken einen oder mehrere Einsatzplätze für Freiwillige an. Unter den Einsatzstellen befinden sich Stadt- und Landesbibliotheken, Bücherhallen, Gemeindebüchereien sowie spezielle Bibliotheken wie z. B. die Heinrich-Böll-Bibliothek in Berlin. Der Anteil der Bibliotheken, die sich auf dem Land oder in einer Kleinstadt befinden, liegt bei 15 Prozent.

Der Einsatz in den Jugendfreiwilligendiensten (bis 26 Jahre) erfolgt in der Regel von September bis zum August des Folgejahres. Der Einsatz auf den generationsoffenen Plätzen für Menschen ab 27 Jahre ist jederzeit möglich und erfolgt abhängig von den Interessensbekundungen der Freiwilligen. Das Anmeldeverfahren für die Jugendfreiwilligendienste findet von Januar bis April über eine zentrale Vermittlungsplattform der $\mathrm{BKJ}^{4}$ statt. Für den Jahrgang 2020/2021 haben sich etwa 700 Personen für

\footnotetext{
bände haben sich in der BKJ zusammengeschlossen. Die Mitglieder der BKJ sind in den Sparten Bildende Kunst, Literatur, Medien, Museum, Literatur, Musik, Spiel, Tanz, Theater und Zirkus aktiv. Sie vertreten unterschiedliche Einrichtungstypen und Praxisformen der Kulturellen Bildung. Alle, die sich in der Jugendarbeit, im Kulturbereich oder der Schule praktisch oder wissenschaftlich mit Kultureller Bildung beschäftigen oder dies planen, berät die BKJ fachlich und strategisch. Sie sorgt für Vernetzung, Dialog und den Austausch von Wissen und Erfahrungen.

Die BKJ unterstützt freiwilliges Engagement in den Bereichen Kultur und Bildung. Sie vermittelt - im Verbund mit Träger-Organisationen in ganz Deutschland - etwa 2500 Plätze in Freiwilligendiensten.

3 Vgl. BKJ (2017a) und BKJ (2017b).

4 www.freiwilligendienste-kultur-bildung.de.
}

einen Platz in einer Bibliothek angemeldet, das sind etwa 20 Prozent aller Interessierten. ${ }^{5}$

\subsection{Tätigkeitsbereiche der Freiwilligen in Bibliotheken}

Die Bibliotheken setzen ihre Freiwilligen in unterschiedlichen Tätigkeitsbereichen ein. Im Folgenden sind beispielhaft einige Aufgabefelder benannt:

- Auspacken und Kontrollieren von Buchlieferungen,

- Einstellen von Medien, Regalordnung, Bücher einsortieren, Etikettierung,

- Mitarbeit in der Ausleihverbuchung und Information,

- technische Medienbearbeitung,

- Mithilfe bei Digitalisierungsprojekten,

- Erstellung von Videotutorials,

- digitale Vorlesestunde für die Facebookseite der Bibliothek,

- Unterstützung bei Klassenführungen und Veranstaltungen für Kinder und Jugendliche,

- Vervollständigung der bibliothekspädagogischen Materialien,

- Betreuung der Technik bei Lesungen und Buchbesprechungen,

- Buchvorstellung für Senior`innen,

- Unterstützung der Buchbinderei,

- PR- und Öffentlichkeitsarbeit (u.a. Webseiten-Pflege, Social Media, Erstellung Flyer).

Im Vergleich mit anderen Kultur- und Bildungsbereichen in den Freiwilligendiensten zeigen Bibliotheken einen hohen Anteil an Sortiertätigkeiten wie z.B. Bücher einordnen oder Daten erfassen (62 vs. 37 Prozent; vgl. Abb. 1). Weitere Tätigkeitsbereiche ähneln denen, die Freiwillige auch in anderen Einsatzstellen übernehmen: So bietet je etwa die Hälfte der Bibliotheken den Freiwilligen die Möglichkeit zur Mitarbeit in der Organisation von Veranstaltungen sowie in der Öffentlichkeits- und Recherchearbeit. Zwei Fünftel der Bibliotheken unterbreiten den Freiwilligen künstlerische oder kreative Tätigkeiten. Die Möglichkeit, mit Medien bzw. Büchern zu arbeiten, findet sich in Bibliotheken nachvollziehbar etwas häufiger als in anderen Einrichtungen. Eher selten werden den Freiwilligen soziale Tätigkeiten angeboten, wie z.B. Personen begleiten und in ihrem Lernen zu unterstützen oder technisch-handwerkliche Tätigkeiten (ein Fünftel und weni-

5 Stand: Mai 2020. 


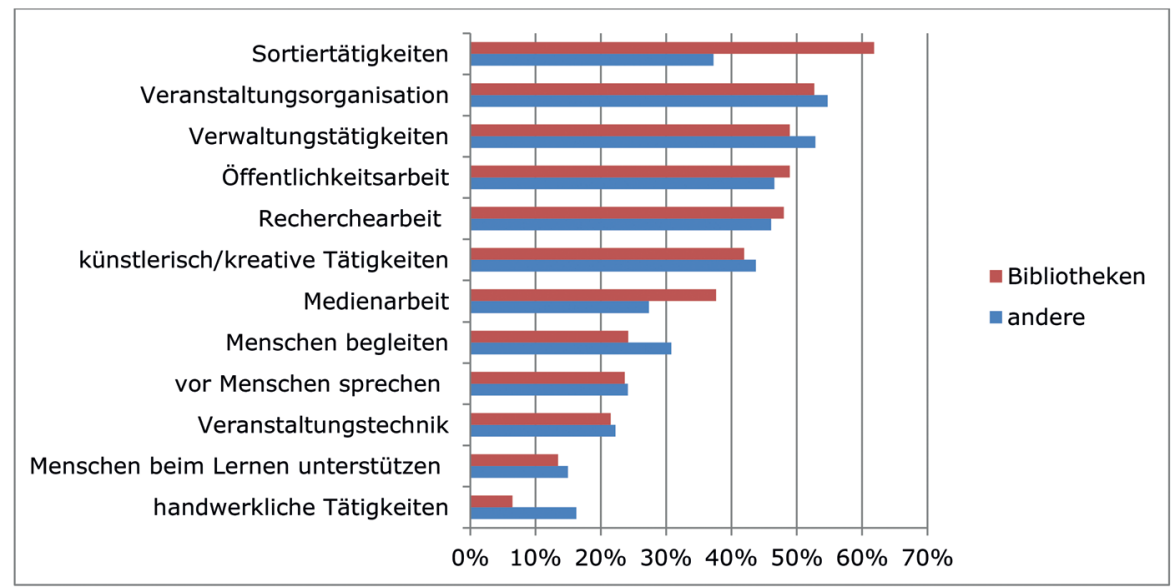

Abb. 1: Tätigkeiten der Freiwilligen ( $\mathrm{N}_{\text {Bibliotheken }}=186, \mathrm{~N}_{\text {andere }}=3149$ (Einsatzplätze)

ger). Doch dass auch diese Tätigkeitsbereiche sich sehr gut als Einsatzbereiche für Freiwillige eignen, zeigen die Bibliotheken, die Freiwilligen diese Tätigkeiten explizit anbieten.

Für den Einsatz der Freiwilligen ist wichtig, dass ihnen die Tätigkeit Spaß macht, sinnvoll und abwechslungsreich ist und dass sie eigene Ideen in die Arbeit einbringen können. Das fördert nachweislich die Zufriedenheit der Freiwilligen mit ihrer Tätigkeit. Außerdem schätzen die Freiwilligen das Gefühl, dass sie einen sozialen bzw. gesellschaftlichen Beitrag leisten. ${ }^{6}$

Was motiviert Bibliotheken, (jungen) Menschen einen Freiwilligenplatz anzubieten und dafür einen finanziellen Beitrag zu leisten? Die folgenden zwei Zitate sollen beispielhaft darauf Antwort geben: ${ }^{7}$

\footnotetext{
„Die Freiwilligen bringen ihre Sichtweise aktiv ein, besonders was Trends angeht und worauf es bei der jungen Generation ankommt.“(2020)

„Es ist ein besonderes Anliegen der Bibliothek, engagierten jungen Leuten Einblicke in ihre vielfältigen Buchbestände zu ermöglichen und sie an den Umgang mit historischen Beständen und Sammlungen heranzuführen. Insgesamt ist das FSJ Kultur ein geschätzter Bestandteil der Bibliotheksarbeit geworden, bringen sich doch die Freiwilligen mit neuen Perspektiven und Ideen ein und sorgen nicht zuletzt regelmäßig für frischen Wind in der Bibliothek."(2020)
}

6 Vgl. BKJ (2017a).

7 Die Zitate entstammen einer Umfrage unter Bibliotheken im Trägerverbund Kultur und Bildung (Mai 2020).

\section{Inklusion und Diversität in den Freiwilligendiensten Kultur und Bildung}

Die Einsatzstellen im Trägerverbund Freiwilligendienste Kultur und Bildung werden von einem (regional) zuständigen Träger anerkannt und müssen spezielle Qualitätsstandards erfüllen. Grundlegendes Qualitätsziel in den Standards ist die Bereitschaft der Einsatzstellen, den gemeinwohlorientierten und arbeitsmarktneutralen Einsatz der Freiwilligen sicherzustellen sowie Diversität und Inklusion in den Freiwilligendiensten zu unterstützen. Mit ihrem Angebot möchte sich die BKJ an alle Personen richten, die einen Freiwilligendienst in einer Kultureinrichtung absolvieren wollen. Keine Person soll aufgrund von Schul- und Berufsabschluss, BeHinderung ${ }^{8}$, Geschlechtsidentität, ökonomischer und sozialer Herkunft oder rassistischen Zuschreibungen benachteiligt werden.

Um diesem inklusiven Ziel Nachdruck zu verleihen, wurde 2014 im Trägerverbund Freiwilligendienste Kultur und Bildung eine Roadmap verabschiedet, die Maßnahmen für eine Öffnung der Dienste und den Abbau von Barrieren auf allen Ebenen und in allen Bereichen festhält. Ausgangspunkt dafür war eine Selbstanalyse mit dem Ergebnis, dass die meisten jungen Freiwilligen in den Freiwilligendiensten Kultur und Bildung zum mehrfachprivilegierten Nachwuchs gehören: Sie sind mehrheitlich weiß, cis, leben ohne BeHinderungen und haben zu über 90 Prozent Abitur. ${ }^{9}$ Damit sind die Freiwilligendienste im Träger-

8 BeHinderung in dieser Schreibweise soll darauf hinweisen, dass Menschen aufgrund ihrer körperlichen oder kognitiven Verfasstheit von gesellschaftlichen Rahmenbedingungen „behindert“ werden. 9 Vgl. Schütze (2017). 
verbund Freiwilligendienste Kultur und Bildung ein Abbild der Institutionen im Bereich Kultur ${ }^{10}$ und von Kulturengagement. Denn die Daten zeigen, dass es für das freiwillige Engagement in und für Kultur zwar gut gelingt, die sogenannte Mittelschicht (Lebensältere, hoher Bildungsabschluss, ökonomisch wohlsituiert) einzubinden, aber weniger gut, gesellschaftlich marginalisierte Gruppen anzusprechen. ${ }^{11}$

Der Schulabschluss kann sehr gut als Ausgangspunkt genutzt werden, um Inklusionsprozesse zu begründen. Über das Schulsystem verankern sich strukturell bereits verschiedene Formen von gesellschaftlicher Diskriminierung. So gilt: Privilegierte Personen erreichen häufig einen formal höheren Bildungsabschluss als nicht-privilegierte, die nach ökonomischen und sozialen Gesichtspunkten bereits benachteiligt sind. Am Schulabschluss wiederum lässt sich festmachen, welche Zugänge und Chancen (junge) Menschen heute haben. ${ }^{12}$

Für non-formales und informelles Lernen, so wie es in den Freiwilligendiensten stattfindet, zeigt sich ebenfalls ein negativer Zusammenhang zwischen formal niedrigen Bildungsabschlüssen und Möglichkeiten weiteren Lernens. ${ }^{13}$ So bieten Freiwilligendienste biografische und berufliche Orientierung und ermöglichen den Erwerb berufsrelevanter, aber auch sozialer Kompetenzen. ${ }^{14}$ Von den Freiwilligen in den Freiwilligendiensten Kultur und Bildung wird diese persönliche Entwicklung und berufsorientierende Wirkung des Freiwilligendienstes als sehr bedeutsam eingeschätzt: ${ }^{15}$ Etwa zwei Drittel der Freiwilligen (bis 26 Jahre) finden, dass ihr Freiwilligendienst zu ihrer beruflichen Orientierung und Entwicklung beigetragen hat, ein weiteres Fünftel bejaht das zumindest zum Teil. Auch ältere Freiwillige (ab 27 Jahre) profitieren beruflich von einem Freiwilligendienst. Denn etwa drei Viertel von ihnen verfolgen nach dem Freiwilligendienst ein berufliches Interesse. So sieht z.B. eine Freiwillige das Besondere an ihrem Freiwilligendienst: „dass ich dadurch [...] in einem völlig neuen Themenfeld tätig geworden bin, das mich zunehmend begeistert“. Und ein anderer: „Ich hatte persönlich und beruflich eine schwere Zeit und war arbeitslos. Meine BFD-Stelle war nicht nur die erste Chance etwas zu machen, was ich schon immer wollte [...]. Ich konnte etwas

10 Vgl. Micossé-Aikins und Sharifi (2017).

11 Vgl. BKJ (2017c), BKJ (2019).

12 Vgl. Bildungsberichterstattung (2018), BMFSFJ (2017a), Allmendinger (2013).

13 Vgl. Bildungsberichterstattung (2018).

14 Vgl. BMFSFJ (2015).

$15 \mathrm{Vgl}$. BKJ (2017a). lernen und meine eigenen Stärken neu entdecken. ${ }^{" 16}$ Deshalb ist es wichtig, dass alle interessierten Personen von einem biografisch so stärkenden und durch öffentliche Förderung möglich gemachten Programm profitieren können und nicht nur Freiwillige, die bereits privilegiert sind. Dafür bedarf es eines genauen Hinsehens und der Sensibilität für strukturelle Barrieren, damit benachteiligende Bildungsbiografien nicht weitergeschrieben werden.

Für den Jahrgang 2020/2021 erhielten etwa 75 Prozent der jungen Freiwilligen mit Abitur einen Platz in einer Bibliothek, etwa zu 25 Prozent Freiwillige mit einem Realschulabschluss, aber kein ${ }^{\star}$ Freiwillige ${ }^{\star}{ }$ mit Hauptschuloder ohne Abschluss. ${ }^{17}$ Damit zeigen Bibliotheken zwar eine größere Durchlässigkeit für Personen ohne Abitur als andere Kultureinsatzstellen. Dennoch bedeutet Diversitätsorientierung auch für Bibliotheken, ihre bisherige Auswahllogik genau zu betrachten und sich zu fragen, welche Personengruppen bzw. Communities in ihrem Haus fehlen. Eine diversitätsbewusste Besetzung im Freiwilligendienst kann dabei einer von vielen Bausteinen auf dem Weg zu einer inklusiven und diskriminierungskritischen Bibliothek sein.

Für die Hälfte der jungen Kultur-Freiwilligen ist ihr Tätigkeitsbereich völlig neu. Damit unterscheiden sie sich von Freiwilligen aus nicht-kulturellen Freiwilligendiensten (36 Prozent vs. 65 Prozent ${ }^{18}$ ). Das Potenzial, dass darin steckt, zeigt sich darin, dass viele Kultur-Freiwillige durch ihren Freiwilligendienst ein für sich attraktives Berufsfeld entdecken. Möchten Bibliotheken also in ihrer Personalpolitik die Einstellung von Personen aus marginalisierten Gruppen gezielt fördern, kann der Freiwilligendienst ein erster Ansatzpunkt sein, um langfristig Zugänge zu den gewünschten Personengruppen zu schaffen. ${ }^{19}$

Vor der Möglichkeit, diversere Personen als bisher auszuwählen, steht aber zuerst die Gewinnung von Personen, die sich überhaupt für einen Einsatzplatz interessieren. Das ist eine zentrale Herausforderung, der sich der Trägerverbund Freiwilligendienste Kultur und Bildung stellen muss. Damit sich diversere Freiwillige als bisher angesprochen fühlen, müssen (neue) Zugänge geschaffen und vorhandene Barrieren konsequent abgebaut werden. In der Zusammenarbeit mit Einsatzstellen bedeutet das z. B., die Öffentlichkeitsarbeit und das Vermittlungsverfahren so zu gestalten, dass sich Personen mit unterschiedlichen Biografien und Fähigkeiten willkommen fühlen, aber auch eine Chance erhalten, für den jeweiligen Platz aus-

$16 \operatorname{BKJ}(2017 b)$.

17 Stand: Mai 2020.

$18 \mathrm{Vgl}$. BKJ (2017a), BMFSFJ (2015).

19 Vgl. Akkoyun et al. (2020). 


\title{
Freiwilligendienste Platzsuche Merkliste Anmeldungen Mein Konto FAQ-Hilfe Kultur und Bildung
}

\author{
Bibliotheksarbeit // Stadtbibliothek „Stadt XYZ”
}

\begin{abstract}
Bei uns kannst du die Funktionen und Aufgaben einer öffentlichen Bibliothek kennenlernen. Hinter den Kulissen der Stadtbibliothek gibt es vielfältige Aufgaben, die Du kennenlernen kannst.

In folgenden Bereichen kannst du bei uns tätig werden:

-Medienbearbeitung: Medien bearbeiten und sortieren, Neu- oder Umorganisieren von Medien, die vorhandenen Medienbestände an die Wünsche und Bedarfe der Nutzer*innen anpassen,

-Kund*innenberatung: Austausch, Beratung und die Kommunikation mit unseren Besucher*innen,

-Veranstaltungsorganisation: Ideenfindung, Organisation und Durchführung von Veranstaltungen für Kinder, Jugendliche oder Erwachsene (z. B. Lesungen, Bilderbuchkino, Leseförderung).

Wenn Du Lust hast, Deine kreativen Ideen bei uns einzubringen geben wir dir gerne die Möglichkeit. Zum Beispiel bei einem eigenen Projekt, bei dem wir dich gerne unterstützen.

Wir freuen uns auf dich!
\end{abstract}

Abb. 2: Tätigkeitsprofil für einen Einsatzplatz in einer Bibliothek

gewählt und gut eingebunden zu werden. Um das zu erreichen, müssen Einsatzstellen, also auch Bibliotheken, zumindest drei Kernkompetenzen mitbringen oder entwickeln: das Schnüren von vielseitigen Aufgabenpaketen, Transparenz schaffen über die Zugänglichkeiten und Barrieren im eigenen Haus sowie die Durchführung eines diversitätsbewussten Vermittlungsverfahrens für alle Interessierten. Darüber hinaus ist die Öffentlichkeitsarbeit der Einsatzstellen vor Ort ein hilfreicher Türöffner, um unterschiedlichste Personen für einen Freiwilligendienst einzuladen.

\subsection{Vielseitige Aufgabenpakete}

Im Trägerverbund Freiwilligendienste Kultur und Bildung werden alle Einsatzplätze über die zentrale Vermittlungsplattform der BKJ veröffentlicht. Mit der Beschreibung ihrer Einsatzplätze gewähren die Einsatzstellen so einen Einblick in das angebotene Einsatzfeld für die zukünftigen Freiwilligen. Ein möglichst breites Aufgabenspektrum bietet hier den Interessent*innen eine Auswahl an Tätigkeiten. Die Aufgabenfelder sollten offen und veränderbar sein, damit sich die Freiwilligen entsprechend ihrer Interessen und Stärken einbringen können.

Als Beispiel für ein aktuelles Bibliotheksangebot, dass unterschiedliche Interessen anspricht, zeigt Abb. 2 ein Tätigkeitsprofil für einen Einsatzplatz, das Veranstaltungsorganisation und unterschiedliche Medienarbeit, aber auch sozial-kommunikative Tätigkeiten umfasst (vgl. Abb. 2). Mit diesem Angebot erreicht die Bibliothek möglicherweise mehr Personen mit unterschiedlichen Interes- sen. In den Kennenlerngesprächen kann die Bibliothek dann gemeinsam mit der interessierten Person schauen, welche Tätigkeiten am besten passen.

\subsection{Transparenz von Zugänglichkeiten und Barrieren}

Barrieren können in verschiedener Form auftreten: räumlich, z.B. durch fehlende rollstuhlgerechte Wege, Aufzüge, Toiletten oder allgemein schlechte Erreichbarkeit, kommunikativ, z.B. aufgrund fehlender Leitsysteme bei Sehbeeinträchtigung oder Einsprachigkeit des Teams, institutionell/sozial, z.B. aufgrund von ausschließenden Selbstverständlichkeiten und Vorurteilen sowie aufgabenbezogen, z. B. durch die Art und Weise, wie Aufgaben in der Einsatzstelle bewältigt werden sollen. ${ }^{20}$ Es existieren häufig viele organisationale Barrieren und nicht alle können trotz inklusiver Grundhaltung von heute auf morgen abgebaut werden. Wichtig ist aber das kontinuierliche Ringen darum und bis dahin die Transparenz über vorhandene Barrieren.

Um Zugänglichkeiten und Barrieren für die einzelnen Einsatzplätze im Trägerverbund Freiwilligendienste Kultur und Bildung transparent $\mathrm{zu}$ machen, bietet die zentrale Vermittlungsplattform der BKJ den Einsatzstellen die Möglichkeit, Angaben zu rollstuhlgerechten Wegen, leichter Orientierung und den Sprachmöglichkeiten im Team anzugeben (vgl. Abb. 3).

20 Vgl. Meyer und Kieslinger (2014). 


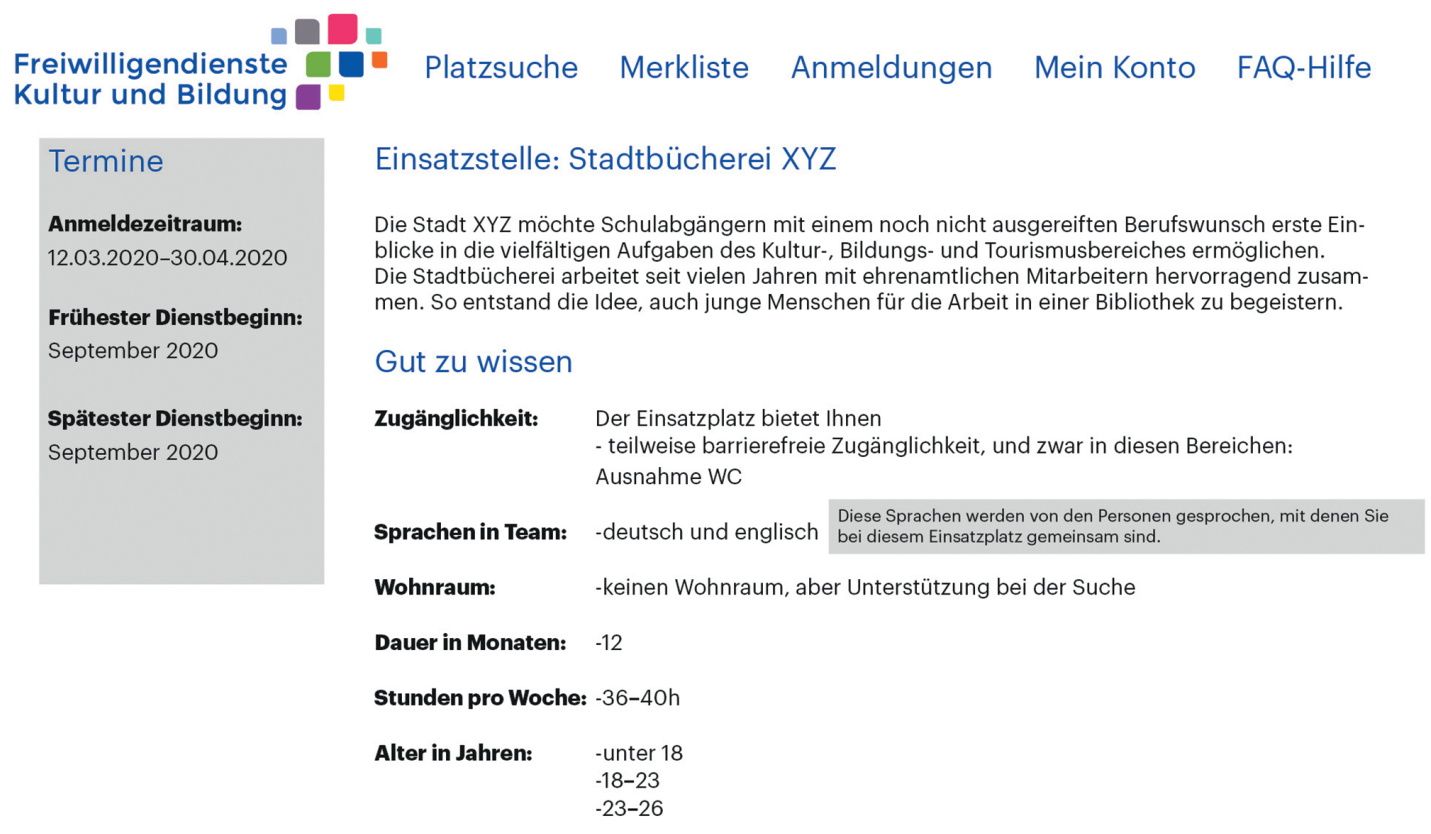

Abb. 3: Tätigkeitsprofil für einen Einsatzplatz in einer Bibliothek

Dass diese Daten sinnvoll sind, beweist auch eine Erhebung von Gleichstellungsdaten, die die BKJ aktuell jedes Jahr bei den Interessent*innen für Einsatzplätze in den Freiwilligendiensten Kultur und Bildung durchführt. Die Personen werden gefragt, ob sie sich aufgrund bestimmter Zugehörigkeiten in Deutschland benachteiligt fühlen. Diese Befragung ist freiwillig und wurde für den Jugendfreiwilligendienstejahrgang 2020/2021 von etwa einem Drittel der 3500 Interessent*innen genutzt. ${ }^{21}$ Fünf Prozent der Befragten geben darin an, dass sie eine (teilweise) BeHinderung haben, die sie in ihrem Leben einschränkt. Für diese Personen können die Angaben über die Zugänglichkeiten und Barrieren von Bibliotheken sehr wichtig sein. Sie helfen den Interessierten einzuschätzen, ob der angebotene Einsatzplatz für sie ein reales Angebot darstellt.

\subsection{Faires Vermittlungsverfahren für alle Interessierten}

Um ein faires Vermittlungsverfahren für alle Interessierten zu gewährleisten, wurde die zentrale Vermittlungsplattform der BKJ so konzipiert, dass Einsatzstellen die persönlichen Anmeldedaten der Interessierten (Alter, Name, Herkunft, Schulabschluss) nicht erfahren. Damit soll verhindert werden, dass unbewusste Vorurteile schon im Vor-

21 Stand: Mai 2020. feld der Kennenlerngespräche die Entscheidung in den Einsatzstellen beeinflussen. ${ }^{22} \mathrm{Zu}$ diesem Prozedere befragt, befanden etwa drei Viertel aller Interessierten in den letzten Jahren eine Anonymisierung als gute Möglichkeit, um Ausschluss im Vorfeld zu verhindern. Als Illustration seien hier drei Äußerungen von Befragten wiedergegeben: ${ }^{23}$

„Ich finde es für die Chancengleichheit gut, dass Alter und Herkunft zuerst nicht offen für die Einsatzstellen ersichtlich sind. So bekommt jeder eine faire Chance.“ (2016)

„Durch die Anonymität von Alter, Herkunft und Bildung wird jeder Bewerber auf das Wichtige reduziert: Die Motivation am FSJ.“(2017)

„Dieses Bewerbungsverfahren ist ein gelungener Versuch, die häufig ungerechten Bewerbungsverfahren fair zu gestalten. Danke!“ (2020)

Das anonymisierte Anmeldeverfahren funktioniert aber nur dann gut, wenn Einsatzstellen tatsächlich offen sind für Personen mit unterschiedlichen Biografien und Fähigkeiten. Das bedeutet beispielsweise auch, Personen zu bevorzugen, die von Diskriminierung betroffen sind (vgl. $\S 5 \mathrm{AGG}$ ). Dient das Kennlerngespräch aber bewusst oder unbewusst dafür, dass Interessent*innen z.B. aufgrund von Alter, Herkunft oder Schulabschluss ungleiche Chan-

22 Vgl. Antidiskriminierungsstelle des Bundes (2020).

23 Diese und folgende Zitate wurden Befragungen entnommen, die von der BKJ von 2016 bis 2020 bei den Interessent ${ }^{\star}$ innen für Einsatzplätze in den Freiwilligendiensten Kultur und Bildung durchgeführt wurden. 
cen erhalten, dann führt das bei den jungen Menschen verständlicherweise zu Frustration.

„Ich hatte das Gefühl, dass ich bei einigen Einsatzstellen einen Nachteil hatte, da ich noch nicht 18 Jahre alt war.“ (2017) „Generell finde ich es gut, wenn nebensächliche Faktoren ausgeschlossen werden und es somit nur auf den Charakter ankommt, aber falls es Vorurteile gibt, können die auch noch bei einem Vorstellungsgespräch subtil mitwirken.“ (2017)

Wird also im Kennenlerngespräch z. B. immer wieder die Person ausgewählt, von der die Einsatzstelle die höchste Leistung erwartet oder die dem bestehenden Personal am ähnlichsten ist, nützt auch ein anonymisiertes Anmeldeverfahren wenig. Für ein faires, und d.h. diversitätsbewusstes Vermittlungsverfahren ist es deshalb wichtig, dass Einsatzstellen sich zusammen mit ihrem Träger austauschen, um mögliche institutionelle bzw. soziale Barrieren vorab zu identifizieren. Das ist die Grundlage, um in der Auswahl von neuen Freiwilligen unfaire Ausschlusskriterien weitestgehend verhindern $\mathrm{zu}$ können.

\subsection{Zielgerichtete Öffentlichkeitsarbeit für Interessierte vor Ort}

Die Einsatzstelle ist mitverantwortlich für die Gewinnung neuer Freiwilliger. Dafür kann sie auf ihrer Webseite und/ oder in ihren sozialen Netzwerken, aber auch im Rahmen ihrer Presse- und Medienarbeit über die Arbeit und die Projekte der Freiwilligen im eigenen Haus berichten. Das erlaubt anderen Interessierten einen Einblick in die möglichen Tätigkeitsbereiche und Rahmenbedingungen des Freiwilligendienstes. Um Inklusion und Diversität zu unterstützen, ist es wichtig, auch Personen, die bisher im Freiwilligendienst unterrepräsentiert sind, zu erreichen. Dafür sind Bibliotheken oft ein besonders geeigneter Ort, da sie von unterschiedlichsten Menschen aufgesucht werden. Gerade junge Menschen ohne Abitur, aber auch lebensältere Personen suchen häufig nach einem Einsatzort in ihrer näheren Umgebung. Die direkte Ansprache oder eine gezielte Ansprache über die eigene Webseite kann hier sehr erfolgreich sein. Um eine Vielfalt von Menschen anzusprechen, ist dafür folgendes zu beachten: ${ }^{24}$

- Informationen sollten in einfacher Sprache formuliert sein, wenn möglich auch in Leichter Sprache. ${ }^{25}$

- Anzustreben ist eine diskriminierungsfreie Sprachund Bildgestaltung sowie gendersensible Sprache.

24 Vgl. auch BAK FSJ (2019).

25 Siehe dazu das Glossar der BKJ unter https://bkj.nu/glossarfwd.
- Unterschiedliche Tätigkeitsbereiche können vorgestellt werden, die zeigen, dass Personen mit unterschiedlichen Fähigkeiten willkommen sind.

- Hilfreich sind Interviews oder kurze Statements von Personen, die bisher im Freiwilligendienst unterrepräsentiert sind: Das können ehemalige Freiwillige, aber auch andere freiwillig Engagierte oder hauptamtliche Mitarbeiter^innen der Einrichtung sein.

- Die Benennung einer Ansprechperson/Ansprechpersonen mit Telefonnummer und/oder E-Mail-Adresse kann die Barriere verringern, Kontakt mit der Einrichtung aufzunehmen.

So lässt sich Inklusion in den Freiwilligendiensten Kultur und Bildung als Recht für alle verstehen, einen Einsatzplatz in den verschiedenen Einrichtungen von Kultur und Bildung erhalten $\mathrm{zu}$ können und sich gut aufgehoben $\mathrm{zu}$ fühlen. Ziel der BKJ ist es, in ihren Freiwilligendiensten die aktuelle gesellschaftliche Realität abzubilden, die geprägt ist durch eine Vielfalt an Menschen mit unterschiedlichen Biografien und Diskriminierungserfahrungen. Die Erreichung eines solchen Ziels setzt einen kritischen Blick auf Strukturen, aber auch auf sich selbst voraus. So bedarf es grundlegend einer gezielten Reflexion der organisationalen Rahmenbedingungen und Gegebenheiten im Trägerverbund und in den Einsatzstellen. Wichtig ist aber auch die Selbstreflexion der Mitarbeiter*innen und Verantwortlichen der Freiwilligendienste über eigene Privilegien und (unbewusste) Anteile an der Erhaltung von ausschließenden Strukturen und Barrieren. Einen inklusiven Weg zu beschreiten bedeutet dann, Strukturen und Prozesse so $\mathrm{zu}$ ändern, dass sich alle Menschen in den Freiwilligendiensten Kultur und Bildung willkommen fühlen.

\section{Mitsprache und Mitbestimmung in den Freiwilligendiensten Kultur und Bildung}

Freiwilliges Engagement, das bedeutet auch die Möglichkeit zur eigenen Mitgestaltung. Deshalb sollten die Orientierung an den Interessen der Freiwilligen und ihre Mitsprache an der Tätigkeit stets in guter Balance zu den Anforderungen in der Einsatzstelle stehen. Die Träger im Verbund Freiwilligendienste Kultur und Bildung haben die Aufgabe, im Gespräch mit den Freiwilligen und Einsatzstellen diesen Partizipationsgedanken immer wieder zu stärken. Die Einsatzstellen sind verantwortlich, die Möglichkeiten zur Mitsprache und Mitgestaltung der Frei- 
willigen in ihren Einrichtungen konkret umzusetzen. Das bedeutet, die Einsatzstelle

- bespricht $\mathrm{zu}$ Beginn des Freiwilligendienstes das Tätigkeitsspektrum mit derdem Freiwilligen und wählt entsprechende Aufgabenpakete zusammen mit der ${ }^{\star}$ dem Freiwilligen aus. Sie orientiert sich dabei an den Interessen und Fähigkeiten der ${ }^{\star}$ des Freiwilligen.

- integriert die ${ }^{\star}$ den Freiwillige ${ }^{\star} \mathrm{n}$ als Mitarbeiter ${ }^{\star}$ in in das jeweilige Team (z.B. durch Teilnahme an Teamsitzungen) und berücksichtigt ihre*seine Anliegen und Meinung.

- beteiligt die ${ }^{\star}$ den Freiwillige ${ }^{\star} \mathrm{n}$ an der konkreten Tages-, Wochen- und Monatsplanung ihres`seines Einsatzes.

- ermöglicht dem ${ }^{\star}$ der Freiwilligen Einblicke in andere Tätigkeitsbereiche innerhalb des eigenen Hauses, z.B. durch Hospitation, und veranschaulicht so demder Freiwilligen verschiedene Berufs- und Engagementfelder.

- führt mit der^dem Freiwilligen in regelmäßigen Abständen ausführliche Gespräche über die Zufriedenheit, Aufgaben, Interessen und Ziele des`der Freiwilligen (Reflexionsgespräche).

- stellt sicher, dass die Freiwilligen mitbestimmen können, welche Schwerpunkte in ihrem Zertifikat aufgenommen werden. ${ }^{26}$

Dass die Mitsprache bei den eigenen Tätigkeiten Freiwilligen wichtig ist, zeigen die folgenden Äußerungen. So hatten aus der Sicht ehemaliger Freiwilliger manche Einsatzstellen „eine sehr starre Vorstellung von den Tätigkeiten der Freiwilligen“. Und sie hätten sich gewünscht, „meine Einsatzstelle auch in anderen Arbeitsbereichen intensiver kennen zu lernen“ oder „dass ich nicht nur eine billige Bürohilfskraft [...], sondern auch in der Kulturarbeit Erfahrungen (hätte) sammeln (können)“. ${ }^{27}$

Freiwillige möchten ihre Ideen und Anregungen, aber auch ihre Fragen und Probleme einbringen können. So betont ein ${ }^{\star}$ e Freiwillige ${ }^{\star}$ r: „Ich hätte mir, bezogen auf meine Einsatzstelle, mehr Möglichkeiten zur Mitsprache gewünscht. Meine Meinung wurde angehört, allerdings schnell wieder vergessen und nicht mehr beachtet.“ Nicht zuletzt sprechen Freiwillige dabei die Frage der Urlaubsund Arbeitszeitenregelung an: „Außerdem wäre es gut,

26 Vgl. Auszug aus den Qualitätsstandards für Einsatzstellen (2020). 27 Alle Befunde und Zitate in diesem Abschnitt stammen aus der BKJ-Gesamtbefragung, die 2015/16 bei allen Freiwilligen in den Freiwilligendiensten Kultur und Bildung durchgeführt wurde, vgl. BKJ (2017a). wenn einem die Urlaubstage und freien Tage nicht vorgeschrieben werden und man da also mitreden kann“.

Die Zufriedenheit der Freiwilligen ist nachweislich dann höher, wenn Freiwillige ernst genommen werden und ausreichend Wertschätzung erhalten, z.B. durch Rückmeldungen zu ihrer Arbeit und durch persönliche Gespräche. ${ }^{28}$ Hier betonen die Freiwilligen die Reflexionsgespräche, die es durch einen größeren zeitlichen Rahmen ermöglichen, sich gemeinsam mit ihnen über ihre Wünsche, Sorgen und Pläne auszutauschen. So erleben Freiwillige es als wichtig und wertschätzend, wenn ihre Interessen und Ideen in der Einsatzstelle berücksichtigt werden. Es zeigt sich, dass die Zufriedenheit der Freiwilligen mit ihrer Begleitung eng mit der Zufriedenheit über ihre Tätigkeiten zusammenhängt: Je besser Freiwillige die Begleitung durch ihre Einsatzstelle einschätzen, umso besser finden sie auch ihre Tätigkeit und umgekehrt. ${ }^{29}$

In einer Gesamtbefragung der BKJ (2017a) unter allen Freiwilligen der Freiwilligendienste Kultur und Bildung wurden aktuelle und ehemalige Freiwillige gefragt, welche Formen der Anerkennung sie sich (noch) gewünscht hätten. Als häufigster Wunsch wurde hier eine bessere Begleitung durch die Einsatzstelle genannt. Dazu gehören aus Sicht der Freiwilligen vor allem eine bessere Kommunikation mit mehr persönlichen Gesprächen und konstruktivem Feedback, aber auch Lob, ein Dankeschön zwischendurch sowie die Wahrnehmung der individuellen Interessen:

„mehr persönliche Gespräche, in denen die allgemeine Bereitschaft, ein FSJ zu absolvieren, mehr gelobt wird. So kam es mir oft so vor, als wäre ich für meine Einsatzstelle etwas ganz Natürliches, (eine Person, die) die gleiche Motivation, nämlich seinen Lebensunterhalt zu bestreiten, habe, wie die anderen Angestellten",

„dass die Leute auch mal herkommen und meine Arbeit sehen und schätzen“,

„vielleicht mal ein kleines ,Dankeschön“ nach einem sehr anstrengenden Tag“.

Freiwillige, die den Freiwilligendienst nach eigener Aussage aufgrund von Problemen mit ihrer Einsatzstelle abgebrochen haben, sind mehrheitlich mit der Begleitung durch ihre Einsatzstelle sowie mit der Wertschätzung und Anerkennung explizit unzufrieden. ${ }^{30}$
28 Vgl. BKJ (2017a). 29 Vgl. ebd. 30 Vgl. BKJ (2017a). 


\subsection{Eigene Projekte der Freiwilligen als Ausdruck für Selbstbestimmung}

Freiwillige in den Freiwilligendiensten Kultur und Bildung erhalten die Möglichkeit, ein eigenes Projekt in der Einsatzstelle durchzuführen. Dafür ermuntern Träger und Einsatzstelle die Freiwilligen am Anfang ihres Dienstes, ein solches Projekt zu übernehmen. Einsatzstellen unterstützen und begleiten die Freiwilligen bei der Umsetzung des Projektes, z. B. bei der Ideenfindung und Planung. Jedes Jahr entstehen so viele spannende kleine und große Projekte, die z.T. auf ganz eigene Ideen der Freiwilligen zurückzuführen sind. Vor allem Freiwillige, die bereits mit der Motivation angetreten sind, eigene Ideen bzw. Projekte in ihrem Freiwilligendienst umzusetzen, erleben die Projektarbeit als einen sehr wichtigen Teil in ihrem Freiwilligendienst. ${ }^{31}$ Für andere, häufig junge Freiwillige kann das Projekt ein Tätigkeitsfeld sein, in dem sie erstmals eigenständig und verantwortungsvoll ihre Ideen umsetzen.

Die Projekte, die Freiwillige durchführen, sind sehr vielseitig. Die folgende Übersicht gibt einen kurzen Einblick in Projekte, die von Freiwilligen in Bibliotheken geplant oder durchgeführt wurden:

- „How to Code“: Coding \& Robotik Workshop in der Stadtbücherei (Nordrhein-Westfalen).

- Planung und Umsetzung einer Kita-Führung mit Biparcour und Bastelstunde (Nordrhein-Westfalen).

- „Superhelden - Marvel, Superman \& Co“: Tauche ein in die Welt der Superhelden und werde kreativ!": Superheld ${ }^{\star}$ innen-Nachmittag mit einem Zeichen-Workshop für Kinder im Alter von 8 bis 12 Jahren (Niedersachsen).

- „Bücherflohmarkt“ der Bücherei anlässlich des Welttages des Buches (Niedersachsen).

- Bilderbuchkino für Kinder: Da die Leidenschaft der Freiwilligen im Bereich der Clownerie liegt, hat sie hierfür eine kleine Inszenierung entwickelt (Niedersachsen).

- Hörspiel: selbst eingesprochen und produziert, das dann in der Bibliothek gehört werden konnte (Bayern).

- „Actionbound-Rallye“ zum Thema Klimawandel: von Freiwilligen konzipiert (Bayern).

- Instagram Account, um die jüngere Zielgruppe besser $\mathrm{zu}$ erreichen: Vorbereitung und Implementierung, Inhalte sind z. B. Zitate aus Büchern, Hinweise auf neue Veröffentlichungen und Buchtipps (Bayern).

31 Vgl. BKJ (2017a).
- Blog-Beitrag über die Erfahrungen als Freiwillige ${ }^{\star}$ r für den Blog der Forschungsbibliothek (Thüringen).

- Ausstellung zu historischen Frauenporträts (Thüringen).

- Lesung: Aufgrund der Coronasituation konnte die Lesung der Stadtbibliothek Grevesmühlen nicht wie geplant stattfinden, sondern nur ohne Publikum, aber die Lesung wurde über den lokalen Fernsehsender ausgestrahlt ${ }^{32}$ (Mecklenburg-Vorpommern)

Die Projekte der Freiwilligen dienen u.a. auch dazu, die Freiwilligen und ihr Engagement nach außen hin sichtbar zu machen. Deshalb ist es eine gute Idee, die Projekte der Freiwilligen auch über die Zeit, in der sie in der Bibliothek tätig sind, hinaus bekannt zu machen und den Freiwilligen für ihre Unterstützung so auch öffentliche Wertschätzung zurückzugeben. Für die Gewinnung neuer Freiwilliger oder auch anderer Helfer^innen kann dies ein wichtiges Signal sein, dass eigene Ideen und Mitgestaltung durch freiwillige Arbeit in der Bibliothek willkommen sind.

\section{Engagementorientierung in den Freiwilligendiensten Kultur und Bildung}

Aus engagementpolitischer Sicht sind Freiwilligendienste durch ihre bildungsbiografische Ausprägung und ihr zeitintensives Engagement interessant. Damit wird begründet, dass sich die Teilnahme an einem Freiwilligendienst als bedeutsam für das weitere freiwillige Engagement einer Person erweisen kann. So können persönliche Erfahrungen und Erlebnisse in einem Freiwilligendienst ein weiteres Engagement im Anschluss an den Freiwilligendienst oder im späteren Leben anregen. ${ }^{33}$ Die Daten aus einer Studie der $\mathrm{BKJ}^{34}$ bestätigen diese Annahme.

Nach ihrem Freiwilligendienst möchten sich 70 Prozent der Freiwilligen weiterhin engagieren. ${ }^{35}$ Eine Befragung ehemaliger Freiwilliger gibt Auskunft darüber, ob diese Absichtserklärung entsprechende Handlungen hervorbringt: Tatsächlich hat sich etwa die Hälfte der ehemaligen Freiwilligen nach ihrem Freiwilligendienst wieder freiwillig engagiert. Dass ihr Freiwilligendienst die Bereitschaft zu weiterem freiwilligen Engagement gestärkt habe,

$32 \mathrm{https} / / /$ youtu.be/I8E2L9mp-jg.

33 Vgl. Simonson et al. (2017).

$34 \mathrm{Vgl}$. BKJ (2017a).

35 Vgl. hier und im Folgenden BKJ (2017a). 
sagt etwa die Hälfte aller Freiwilligen, ein weiteres Drittel stimmt dem zum Teil zu (vgl. Abb. 4).

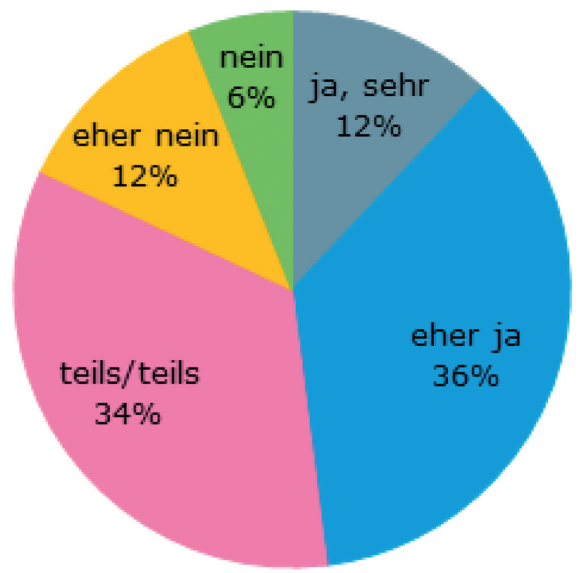

Abb. 4: Hat der Freiwilligendienst ihre Bereitschaft zum freiwilligen Engagement geweckt bzw. gestärkt? ( $n=1515$, Freiwillige bis 26 Jahre)

Knapp ein Viertel der Menschen, die aktuell engagiert sind und in der Vergangenheit einen Freiwilligendienst geleistet haben, nennen diesen als Anstoß für ihr Engagement. ${ }^{36}$ Am häufigsten wird von Engagierten mit Haupt- und Realschulabschluss darauf verwiesen. Die Autor^innen des Freiwilligensurvey 2014 deuten dieses Ergebnis so: „Soziale Ungleichheit im Engagement, über den gesamten Lebenslauf betrachtet, (kann) also möglicherweise verringert" werden, wenn mehr Personen mit Haupt- und Realschulabschlüssen für einen Freiwilligendienst geworben werden können. ${ }^{37}$

Freiwilligendienste sind manchmal für die Freiwilligen die erste Engagement-Erfahrung. In den Freiwilligendiensten der BKJ geben etwa zwei Fünftel der Freiwilligen an, sich vor ihrem Freiwilligendienst noch nie freiwillig engagiert zu haben. Diese Freiwilligen betonen die engagementorientierende Wirkung des Freiwilligendienstes im Nachhinein am stärksten. So kann der Freiwilligendienst einen Zugang zu freiwilligem Engagement darstellen. In engem Zusammenhang damit zeigt sich, dass die Freiwilligen am Ende ihres Freiwilligendienstes stärker als früher bereit sind, Verantwortung für andere $\mathrm{zu}$ übernehmen (69 Prozent), mehr Interesse an gesellschaftlichen Zusammenhängen entwickelt haben (56 Prozent) sowie mehr darüber erfahren haben, wie sie ihr Umfeld mitgestalten können (55 Prozent). ${ }^{38}$
Wichtig für das Interesse an weiterem Engagement und gesellschaftlicher Verantwortung ist, dass der Engagementgedanke für die Freiwilligen im eigenen Freiwilligendienst sichtbar wird. Das bedeutet zum einen, dass Freiwillige das Gefühl haben müssen, mit ihrem Freiwilligendienst einen sozialen bzw. gesellschaftlichen Beitrag zu leisten. Zum anderen ist der wertschätzende Umgang mit den Freiwilligen in den Einsatzstellen wichtig, damit Freiwillige den Freiwilligendienst als eine Form von Engagement erleben und nicht als eine Art Praktikum oder Niedriglohnarbeit. Mitbestimmung der Freiwilligen und Orientierung an ihren Interessen sind hier bewährte Prinzipien. ${ }^{39}$

Auch Bibliotheken können von dem zukünftigen Engagement der Freiwilligen profitieren. Viele junge Menschen verlassen zwar nach dem Freiwilligendienst die Region, in der sie tätig waren. Dennoch geben etwa zwei Fünftel der Einsatzstellen an, dass ehemalige Freiwillige sich weiterhin in ihrer Einrichtung engagieren. Freiwillige, die in ihrem Freiwilligendienst älter als 26 Jahre alt waren, engagieren sich noch häufiger nach ihrem Freiwilligendienst in ihrer ehemaligen Einsatzstelle weiter (etwa die Hälfte $\left.{ }^{40}\right)$.

\section{Trägerarbeit und Ausblick}

Die Arbeit der Träger ist ein wichtiger Bestandteil im Freiwilligendienst. Dazu gehört die individuelle Begleitung der Freiwilligen - vom Beratungs- und Vermittlungsverfahren über die konkrete Begleitung der Freiwilligen während des Freiwilligendienstes, die Ausgestaltung des Bildungsangebots bis hin zur Ehemaligenarbeit. Die Seminar- und Bildungsarbeit der Träger zeigt sich dabei für alle Wirkungsdimensionen des Freiwilligendienstes als bedeutsam: für die persönlichkeits- und gesellschaftsbezogene Entwicklung der Freiwilligen, für die kulturelle Bedeutung sowie die berufs- als auch engagementorientierende Wirkung des Freiwilligendienstes..$^{41}$ Bildungstage begleiten und vervollständigen die Zeit im Freiwilligendienst. Freiwillige werden hier zum Austausch, zu kreativer Arbeit und zum inhaltlichen Diskurs eingeladen. Die Trägerarbeit stellt zudem einen vermittelnden Aspekt im Freiwilligendienst dar, da diese sowohl die Arbeit mit Freiwilligen als auch mit Einsatzstellen umfasst. 
Die Träger stehen in einem engen und kontinuierlichen Austausch mit den Einsatzstellen. Sie beraten die Einsatzstellen in Fragen der Partizipation und guten Begleitung von Freiwilligen sowie zum Thema Anerkennung und Wertschätzung der Freiwilligen. Gerade diese Aspekte zeigen sich zentral für die Begleitung der Freiwilligen und beeinflussen nachweislich die Engagementorientierung der Freiwilligen positiv. ${ }^{42}$

Freiwillige sind mit ihrem Freiwilligendienst zufrieden, wenn sie ihren Einsatz in der Einsatzstelle als sinnvoll erleben, sich durch die Einsatzstelle gut begleitet fühlen und mit ihren Mitsprachemöglichkeiten zufrieden sind. Deshalb bedarf die Arbeit mit Freiwilligen einer kontinuierlichen Reflexion, ob diese Kriterien erfüllt sind. Träger bieten mit ihrer externen Begleitung und Seminararbeit den Freiwilligen - zusätzlich zur Begleitung der Einsatzstellen - dafür passende Räume und Methoden.

Freiwilligendienste sind Lernfelder und bieten Menschen ein non-formales Setting, das ihnen durch Freude an einer selbstgewählten Tätigkeit persönliche Entwicklung, Selbstwirksamkeitserleben, biografische Orientierung und neue Netzwerke bietet. Großartige Möglichkeiten, die in den Freiwilligendiensten Kultur und Bildung bisher vor allem Personen mit Abitur offeriert werden. Ein biografisch so stärkendes Programm sollte aber gerade auch Menschen zugänglich sein, die von gesellschaftlicher Marginalisierung betroffen sind. Der Trägerverbund Freiwilligendienste Kultur und Bildung setzt sich deshalb seit einigen Jahren für eine inklusive Öffnung seiner Freiwilligendienste ein. Zusammen mit ihren Trägern und Einsatzstellen hat sich die BKJ zum Ziel gesetzt, Ausschlüsse und Barrieren aufzudecken, die Menschen daran hindern, einen Freiwilligendienst in einer Kulturund Bildungseinrichtung des Trägerverbunds $\mathrm{zu}$ absolvieren. Bibliotheken als langjährige oder auch als neue Einsatzstellen in den Freiwilligendiensten Kultur und Bildung sind herzlich willkommen, an diesem herausfordernden und sinnstiftenden Prozess mitzuwirken und dafür zu sorgen, dass alle interessierten Personen eine Chance auf einen Einsatzplatz in ihren Einrichtungen erhalten können.

\section{Literaturverzeichnis}

Akkoyun, Yasemin; Ali Bakhsh Naini, Sophie; Boitel, Sophie; Eder, Angelika; Neumeister, Stefanie; Prabha Nising, Lena; Zosik,
Anna (2020): Diversitätsorientierte Nachwuchsförderung und Personalgewinnung im Kunst- und Kulturbereich. Erfahrungen der Stiftung Genshagen und ein Leitfaden für Kulturinstitutionen. Verfügbar unter http://www.stiftung-genshagen.de/filead min/Dateien/2020_Dateien/KUKU/KIWit_Broschuere/KIWit_Br oschuere_Diversitaet.pdf.

Allmendinger, Jutta (2013): Bildungsgesellschaft. Verfügbar unter http://www.bpb.de/gesellschaft/bildung/zukunft-bildung/158 109/teilhabe-durch-bildung.

Antidiskriminierungsstelle des Bundes (2020): Verfügbar unter http://www.antidiskriminierungsstelle.de/SharedDocs/Downlo ads/DE/publikationen/BT_Bericht/Gemeinsamer_Bericht_dritt er_2017.pdf?_blob=publicationFile \&v=12.

BAK FSJ (2019): Arbeitsgruppe Inklusion und Diversität in den Freiwilligendiensten im Bundesarbeitskreis FSJ. Inklusion und Diversität in den Freiwilligendiensten. Erfahrungen und Handlungsempfehlungen. Ein Praxisleitfaden. Berlin.

Bildungsberichterstattung (2018): Bildung in Deutschland 2018.

BMFSFJ (Hrsg.) (2015): Abschlussbericht der gemeinsamen Evaluation des Gesetztes über den Bundesfreiwilligendienst (BFDG) und des Gesetzes zur Förderung von Jugendfreiwilligendiensten (JFDG). Berlin.

BMFSFJ (Hrsg.) (2017): 15. Kinder- und Jugendbericht: „Jugend ermöglichen".

BKJ (2017a): Bundesvereinigung Kulturelle Kinder- und Jugendbildung e.V. Gesamtevaluation aller Jugendfreiwilligendienste im Trägerverbund Freiwilligendienste Kultur und Bildung der BKJ 2015/ 2016. Befragung von Freiwilligen, Einsatzstellen und Alumni. Internes Papier. Berlin.

BKJ (2017b): Bundesvereinigung Kulturelle Kinder- und Jugendbildung e.V. Gesamtevaluation des Bundesfreiwilligendienst Kultur und Bildung der BKJ 2015-2017. Befragung von Freiwilligen, Einsatzstellen und Alumni. Internes Papier. Berlin.

BKJ (2017c): Bundesvereinigung Kulturelle Kinder- und Jugendbildung e.V. Freiwilliges Engagement in Kultur. Sonderauswertung des Freiwilligensurveys 2014. Berlin. Verfügbar unter https://www.bkj.de/publikation/freiwilliges-engagement-inkultur/.

BKJ (2019): Bundesvereinigung Kulturelle Kinder- und Jugendbildung e. V. Kulturvereine. Selbstverständnis, Strukturen, freiwilliges Engagement. In: Kooperation mit ZiviZ (Zivilgesellschaft in Zahlen) gGmbH im Stifterverband. Berlin. Verfügbar unter https://www.bkj.de/publikation/kulturvereine/.

Meyer, Thomas; Kieslinger, Christina (2014): Index für die Jugendarbeit. Stuttgart.

Micossé-Aikins, Sandrine; Sharifi, Bahareh (2017): Kulturinstitutionen ohne Grenzen? Annäherung an einen diskriminierungskritischen Kulturbereich. In: Weiße Flecken. Diskurse und Gedanken über Diskriminierung, Diversität und Inklusion in der Kulturellen Bildung, hg. v. Anja Schütze und Jens Maedler. kopaed: München.

Schütze, Anja (2017): Durch dicke Bretter bohren. In: Weiße Flecken. Diskurse und Gedanken über Diskriminierung, Diversität und Inklusion in der Kulturellen Bildung, hg. v. Anja Schütze und Jens Maedler. kopaed: München.

Simonson, Julia; Vogel, Claudia; Tesch-Römer, Clemens (Hrsg.) (2017): Freiwilliges Engagement in Deutschland. Der Deutsche Freiwilligensurvey 2014. Wiesbaden. 

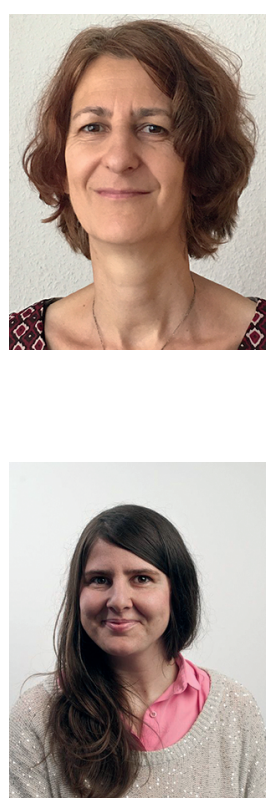

\section{Maud Krohn}

Bundesvereinigung Kulturelle Kinder- und Jugendbildung e.V. (BKJ)

Freiwilliges Engagement und Ehrenamt

Freiwilligendienste Kultur und Bildung

Greifswalder Str. 4

D-10405 Berlin

krohn@bkj.de

\section{Anja Schütze}

Bundesvereinigung Kulturelle Kinder- und Jugendbildung e.V. (BKJ)

Freiwilliges Engagement und Ehrenamt

Freiwilligendienste Kultur und Bildung

Greifswalder Str. 4

D-10405 Berlin

schuetze@bkj.de 